\title{
Modelo de un Personaje en un Entorno Virtual Inteligente
}

\author{
Sandra P. Mateus ${ }^{(1)}$ y John W. Branco ${ }^{(2)}$ \\ (1) Politécnico Colombiano JIC, Facultad de Ingenierías, Cra 48 N 7-151 Of: P19-146, \\ Medellín-Colombia (e-mail: spmateus@elpoli.edu.co). \\ (2) Universidad Nacional de Colombia, Facultad de Minas, Sede Medellín. Carrera 80 No 65-223, \\ Of: M8A-307, Medellín-Colombia (e-mail: jwbranch@unal.edu.co).
}

Recibido Ago. 09, 2011; Aceptado Oct. 05, 2011; Versión final recibida Dic. 21, 2011

\begin{abstract}
Resumen
Este artículo propone un modelo de referencia de un personaje, con base en su percepción y razonamiento, con el fin de alcanzar un realismo visual en un Entorno Virtual Inteligente (EVI). Las etapas propuestas para llegar a la obtención del modelo son: i) caracterización de objetos y el personaje de un EVI; ii) diseño del modelo del EVI; iii) especificación de la técnica de Inteligencia Artificial (IA); iv) definición del nivel de física y creación del sistema semántico para la integración del personaje con el entorno; v) validación del modelo propuesto mediante un prototipo; y vi) evaluación de resultados. El modelo propuesto permite generar un dinamismo adecuado entre el personaje y los elementos de un entorno virtual, requerido en diversas aplicaciones.
\end{abstract}

Palabras clave: entorno virtual inteligente (EVI), personaje virtual, inteligencia artificial

\section{Character Model in an Intelligent Virtual Environment}

\begin{abstract}
In this paper, a character reference model, based on its perception and reasoning, with the objective of achieving visual realism in an Intelligent Virtual Environment (IVE) is proposed. The necessary steps for obtaining the model are: i) to identify the characteristics of the objects and of the virtual character of the IVE; ii) design of the IVE model; iii) specification of the Artificial Intelligence technique (AI); iv) defining the physical level and semantic system for the integration of the character with the environment; $v$ ) validation of the proposed model in a prototype; and vi) evaluation of the results. The proposed model allows generating an appropriate dynamics between the character en the elements of the virtual environment, which is required in several applications.
\end{abstract}

Keywords: intelligent virtual environment (IVE), virtual character, artificial intelligence 


\section{INTRODUCCIÓN}

Los entornos virtuales inteligentes $(E \mathrm{VI})$ están compuestos por la unión de dos importantes áreas de la computación: la realidad virtual y la inteligencia artificial, las cuales tienen múltiples aplicaciones que les permite ser ampliamente utilizadas en campos como la industria, la educación y la investigación, entre otras; además, su acción conjunta les permite alcanzar un completo nivel de aplicabilidad en diversas áreas. La realidad virtual se conoce como la tecnología computacional que simula un ambiente tridimensional que en su mayoría son experiencias visuales en una pantalla o en un dispositivo estereoscópico que en algunas ocasiones incluyen sensaciones auditivas; cuando a un entorno virtual se le incorpora la inteligencia artificial alcanza una nueva característica dando como resultado un sistema de distribución de eventos de forma asincrónica la cual es la que lo diferencia de un AV convencional y de ahí su virtud de alta aplicabilidad (Lozano y Calderón, 2004).

En la actualidad, la mayoría de imágenes que se pueden observar en los principales medios de comunicación contienen elementos que son producto de la realidad virtual, como por ejemplo en los comerciales en los cuales no todos los personajes son actores, o en los documentales donde muestran todos los ángulos de estructuras o seres, o más específicamente, las películas animadas; otra muestra más concreta y clara son los videojuegos que dejan en promedio más de 7 billones de dólares de ganancias al año, y si a todo eso se le agrega IA, se podría alcanzar un nivel de aplicabilidad más amplio, obteniendo un producto capaz de adaptarse a múltiples objetivos.

Las limitaciones en un EVI se observan en el realismo que se obtiene, y esto recae a su vez, en el comportamiento e interacción con los elementos de un EV, realismo necesario en aplicaciones de interés científico de tipo militar, entrenamientos en la medicina, en la herencia cultural, entre otras. Por todo lo anterior, se propone encontrar un modelo, que aplicado al comportamiento de los objetos y agentes de un EVI, permita encontrar un adecuado realismo visual. Este artículo está organizado de la siguiente manera: En la sección siguiente se presenta el marco conceptual alrededor de los entornos virtuales; posteriormente, se detalla la revisión de la literatura; luego, se expone la propuesta la cual incluye el planteamiento del problemay la metodología a utilizar para resolver el problema; finalmente se exponen las conclusiones y se detallan las referencias.

\section{ENTORNOS VIRTUALES}

Independientemente del entorno interesado en su modelización virtual, desde un punto de vista exclusivamente computacional, los entornos virtuales están compuestos por tres modelos que conforman la aplicación informática en tiempo real: un modelo geométrico, un modelo comportamental (en tiempo de ejecución) y un modelo de interacción con el usuario (Lozano y Calderón, 2004).

Modelo geométrico: Es el encargado de atender al bajo nivel gráfico, donde por un lado, los distintos tipos de formatos gráficos empleados, junto con el modelo interno utilizado para el lanzamiento de las ordenes de dibujado o render (grafo de escena, ordenación de primitivas gráficas o displaylists, etc), resumen las propiedades elementales para la visualización de cualquier Entorno Virtual. Tradicionalmente, estos entornos son descritos con base en la colección de primitivas poligonales, líneas, texto, superficies, etc., que constituyen la información espacial visualizada en $3 \mathrm{~d}$.

Modelo Comportamental: Este modelo trata el comportamiento del entorno virtual como aplicación informática en tiempo de ejecución, es decir, atiende al comportamiento dinámico (cambios) de todos los objetos $3 \mathrm{~d}$ que el Entorno Virtual contiene. Un comportamiento considerado, típicamente se refiere al mantenimiento en tiempo de ejecución de propiedades elementales, como posición, orientación, color, etc. de todo elemento (objeto u actor) situado en el entorno virtual; el otro, sería el comportamiento autónomo típicamente asociado a la toma de decisiones de distintos EVI, u objetos autónomos que pueden formar parte de la simulación. 
Interacción con el usuario: Una de las principales expectativas generadas en la mayoría de entornos virtuales, es la libre interacción con los elementos $3 \mathrm{~d}$ del entorno; la inclusión del usuario en el grafo de escena limita de nuevo el modo de interacción con el resto de nodos del grafo, es decir, con su entorno $3 d$.

\section{Entornos Virtuales Inteligentes (EVI)}

El énfasis de los EVI (Aylett y Luck, 2000) reside en incrementar las capacidades comportamentales e interactivas de los entornos virtuales clásicos. Esto se consigue mediante la incorporación de técnicas de IA situadas e interactuando con el sistema gráfico. Aunque existen diferentes técnicas de animación (ej: interpoladores VRML o nodos de secuencia de OpenGLPerformer) y sencillos modelos comportamentales que pueden ayudar a dotar de cierto dinamismo a los entornos clásicos, en la mayoría de ocasiones, su naturaleza de guión o plan precompilado (off-line) sólo logrará mantener el interés del usuario durante un tiempo bastante limitado. Según (Lozano y Calderón, 2004) hay tres formas de incrementar las capacidades interactivas de un entorno virtual se darían mediante la integración de sistemas de IA: Incremento de la actividad del Entorno Virtual: Esto se puede lograr mediante la integración de motores de razonamiento simbólico que interpreten, por ejemplo, las acciones del usuario;Necesidad de una representación del conocimiento; y Alternativa a la simulación física.

La evolución natural de los EVI, pasa por proporcionar entornos de simulación adecuados a mundos $3 \mathrm{~d}$ de naturaleza cambiante, y en este sentido, los entornos dirigidos por eventos proporcionan actualmente un marco adecuado para la investigación y desarrollo en EVI. Los modelos comportamentales y de comunicación clásicos, no satisfacen suficientemente estos requerimientos, debido principalmente a su alta dependencia con el modelo geométrico (grafos de escena), el cual está claramente orientado a una rápida visualización centrada en el usuario, dejando de lado importantes cuestiones comportamentales y de interacción. Finalmente, la integración de EVI, es un problema raramente tratado desde el punto de vista del modelo computacional que soporta el entorno virtual. Dado que estos entornos están llamados ser los entornos de simulación de distintos tipos de EVI, deberán ser capaces de gestionar los nuevos requerimientos dinámicos impuestos. En este sentido, los modelos basados en el envío de recepción asíncrona de eventos proporcionan un modelo de simulación adecuado para la actual intersección entre la IA y los gráficos $3 \mathrm{~d}$ en tiempo real.

\section{REVISIÓN DE LA LITERATURA}

Uno de los primeros EVI es el sistema de programación "Oz" dentro del software DIVE VR (Axling et al., 1996). Oz soportaba la programación basada en reglas, la cual era usada como un lenguaje de programación de alto nivel para la mejora del desempeño en un entorno virtual. Posteriormente Codognet (1999), desarrolló un paquete genérico basado en reglas llamado VRCC, el cual se integraba en VRML y se usaba para definir el comportamiento de agentes a través de reglas. Ambos sistemas dependían de la Programación Lógica basada en Reglas CLP (Constraint Logic Programming). De ahí, fueron surgiendo otras aproximaciones de IA, tales como el ajuste heurístico y la búsqueda local, las cuales son alternativas para mecanismos básicos usados en programación basada en reglas, como lo realizó Codognet (2001), el cual afirmaba que el ajuste heurístico es un buena solución y puede proveer una rápida solución cuando una solución predefinida era perturbada por la acción de un simple objeto. Calderón y Cavazza (2001), desarrollaron un EVI usando GNU Prolog y el motor de juego UnrealTournament. La IA adquiere gran relevancia en la personificación y también en la robótica, sin embargo, la perspectiva gráfica es de gran importancia en figuras animadas. De ahí que se utilicen los Agentes virtuales Inteligentes, los cuales pueden definirse como una entidad de software que basándose en su propio conocimiento del entorno, realiza una serie de operaciones destinadas a realizar un objetivo ya sea por iniciativa propia o porque dada una situación se lo requiera.

Jia y Zhenjiang (2007) desarrollaron la Plataforma "Paladin" (Ver Fig. 1), la cual utiliza agentes colaborativos y una técnica poco utilizada en los IVE como es la Neuroevolución, la cual en este trabajo, combina las Redes Backpropagation con los Algoritmos Evolutivos, lo que crea una 
metodología aceptable. Sin embargo, el entorno de este trabajo es en 2D y la colaboración de los agentes es limitada. En esta misma línea, Mang-Xian y Hai-ming (2008), en su trabajo utilizan agentes colaborativos como un modelo de grupos de agentes virtuales, utilizando el protocolo KQML. Los problemas que muestran los autores utilizando esta metodología, son el tiempo computacional, el control de los objetos y la autonomía de los agentes.

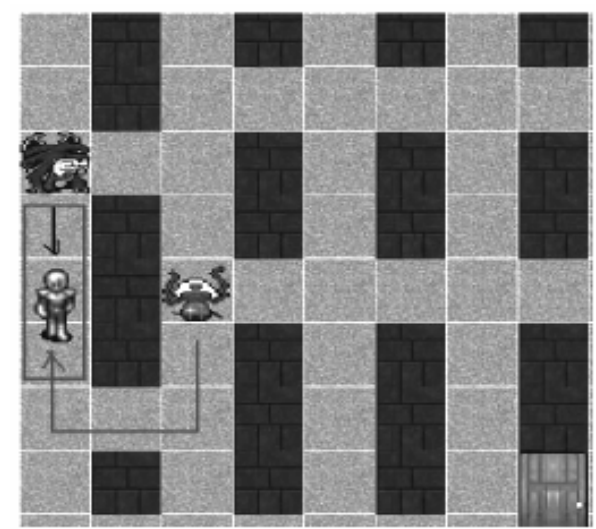

Fig. 1. Plataforma Paladin(Jia y Zhenjiang, 2007)

Mascarenhas et al. (2009), trabajan lo que denominan Agentes Sociales Inteligentes (Ver Fig.2), para identificar diferencias en dos culturas. Ellos de forma general, utilizan una tupla de cinco elementos, que resume las características esenciales en sus agentes. La tupla es <T,R,C,S,O $>$ : ritual, rol, contenido, conjunto de pasos, restricciones. Sin embargo, este trabajo se ve limitado a la integración de una cultura adicional y se ve afectado por la influencia de cada componente.

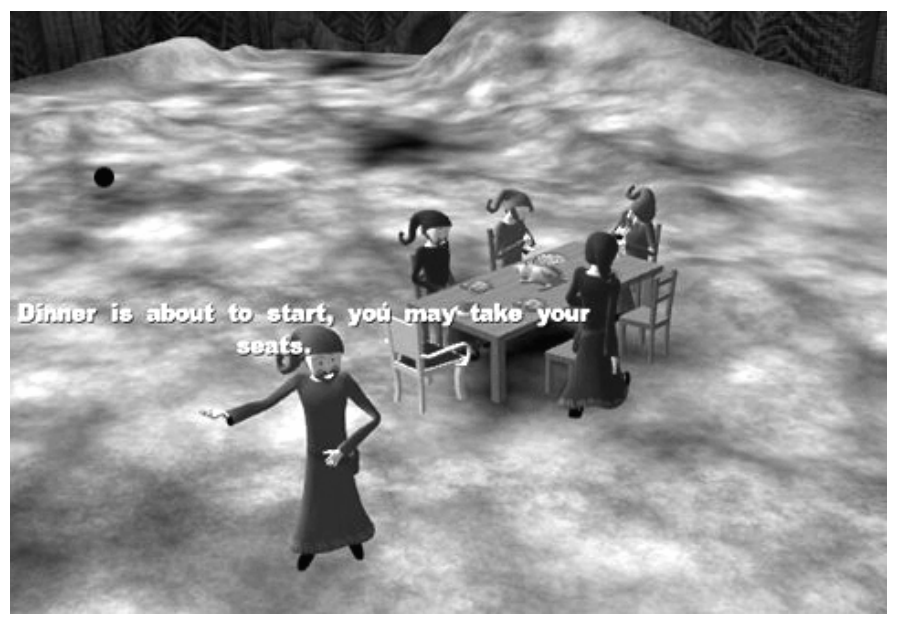

Fig.2. Agentes Sociales Inteligentes (Mascarenhas et al, 2009)

Siguiendo con los agentes, el artículo de Luzardo y Hernández (2007), es una buena introducción a la temática de los EVI y a lo que se denomina los Humanos Virtuales Autónomos, sin embargo en este trabajo no se específica ningún modelo. DosSantos y Osorio (2004) trabajan con Agentes Virtuales Inteligentes orientados hacia la enseñanza - aprendizaje, basados en reglas simples y con navegación asistida y poca recuperación de información. Desde otro enfoque, Tutenel y Bidarra (2008) aplican la Semántica en Juegos y simulaciones (Ver Fig.3), lo que les permite crear una ontología robusta para la generación automática del mundo virtual, pese a que no incorpora Planificación. En esta misma línea Bonis et al. (2008) desarrollaron una plataforma para museos virtuales, con una descripción semántica del contenido para interactuar con el museo, pero al igual que el anterior, no utilizan técnicas de IA. 

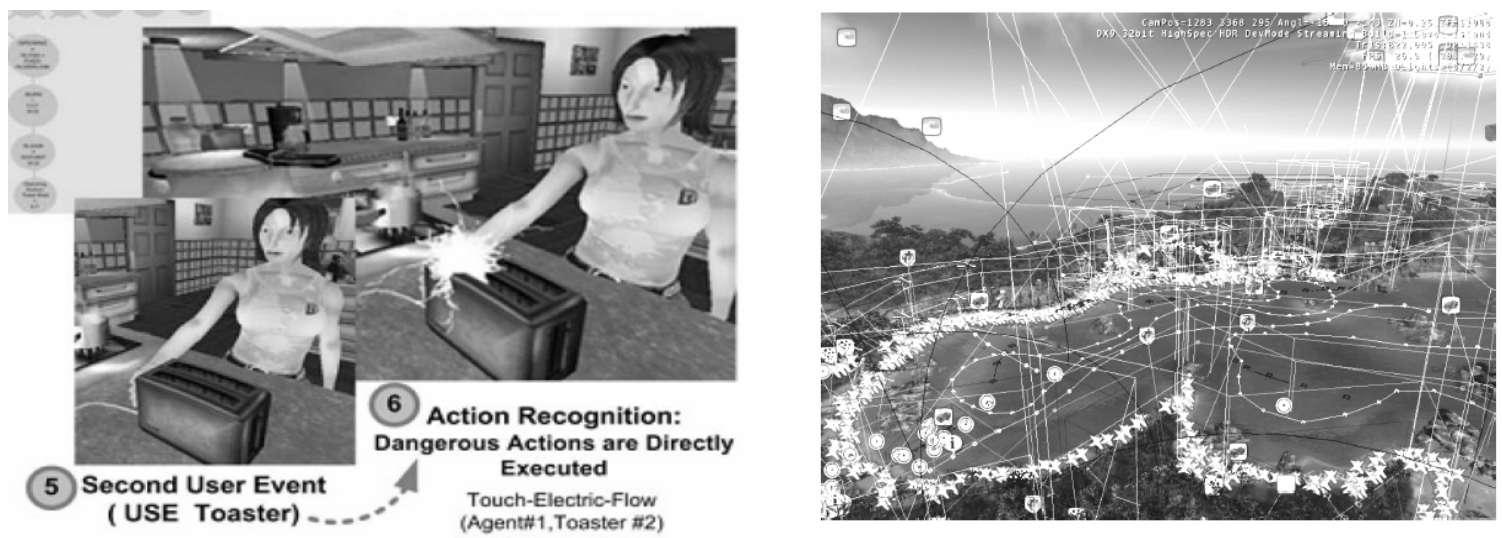

Fig.3. Semántica aplicada en Juegos y Simulaciones Tuteniel y Bidarra(2008)

Ya empleando técnicas de IA, Latoschik y Fröhlich (2007) utilizan reflexión semántica para un IVE, utilizando un modelo por capas, incorporándola así en agentes inteligentes, aunque no muestran una metodología clara en la aplicación de los IVE.

Al mismo tiempo, fueron apareciendo personajes sintéticos y humanos virtuales en los EVI, en los cuales el papel de la IA ha sido fundamental. Algunos ejemplos de personajes (Aylett y Cavazza, 2001) se pueden observar en: personajes no humanos, mecánicos, ficticios como Creatures,Woggles o Teletubbies; también animales como pájaros, pescados, delfines o perros; y humanos virtuales como Marilyn y agentes pedagógicos. A nivel de personajes en los EVI, Decker et al. (2009) proponen una técnica de interacción basada en reconocimiento de discurso emocional (Ver Fig. 4), en la cual permiten que el usuario tome parte en un diálogo con actores virtuales sin restricciones en estilo o expresividad. Las limitaciones de esta técnica, se encuentran en la definición de las categorías emocionales y el hecho de que su impacto depende de las consideraciones del género que definen, como el número de elementos que manejan.

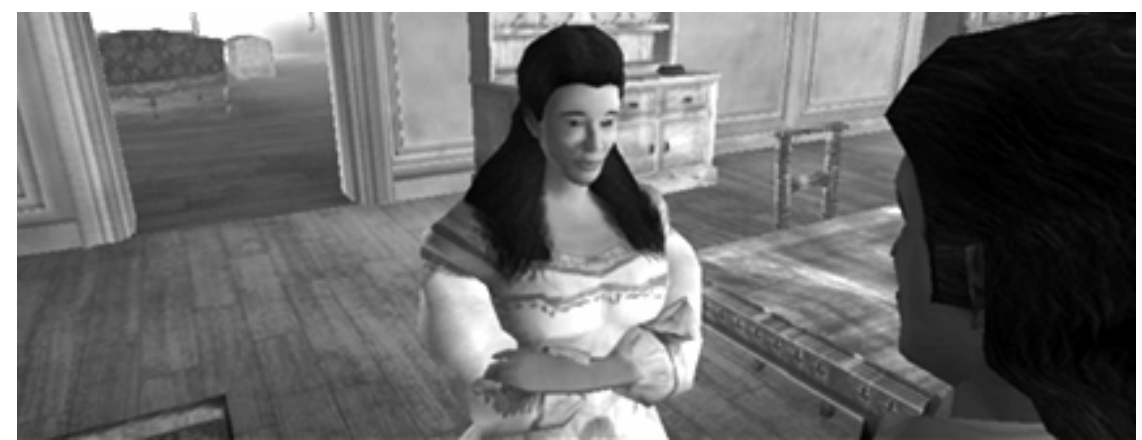

Fig. 4. Discurso emocional (Decker et al., 2009)

Bee et al. (2010), integran la mirada de usuarios en un sistema de narrativa para permitir interacciones entre los personajes. En este trabajo, identifican dos grupos de usuarios: uno que está permanentemente con mirada fija al personaje y la otra muestra un comportamiento de mirada natural entre los ojos de los personajes, las caras y la escena; en el primero no hay una interactividad como tal, pero se mantiene la mirada en el personaje, mientras que en el segundo, se muestran diferentes comportamientos de miradas dependiendo de cómo el personaje esté interactuando. Los autores concluyen con que en una reunión social, no se dan diferencias significativas entre un grupo y el otro.

Siguiendo con el desarrollo de personajes basados en agentes inteligentes como técnica de IA utilizada, Edward et al. (2009) proponen un modelo cognitivo para un personaje. Guo et al. (2009) plantean la optimización del comportamiento del personaje basado en análisis exploratorio. Zhong y Guoxin (2009), presentan el comportamiento de un personaje basado en características grupales. Silveira et al. (2010) desarrollan un método llamado BVP (Problemas basados en valor de banderas) para controlar el personaje. Y finalmente, Gongora e Irvine (2010), desarrollan un 
método para el control del personaje basado en el modelo de agente BDI (creencias, deseos e intenciones), además usando Máquinas de Estado Finito.

La mayoría de trabajos de EVI's, desarrollados y reportados en la literatura hasta el momento, se han visto limitados por un modelo, que aplicado al comportamiento de los objetos y agentes de un $\mathrm{EVI}$, permita encontrar un adecuado realismo visual. Este realismo, está basado en los elementos que permiten un desempeño inteligente tales como: la percepción, el aprendizaje, la comunicación mediante un lenguaje natural y el razonamiento.

Todo lo anterior, así sea visto en forma individual, debe tener un proceso de planificación, para coordinar los niveles de control y animación en personajes, que se encuentra limitado en la literatura. Con base en lo anterior, el desempeño inteligente se centra en determinar la mejor secuencia de acciones a ser ejecutadas en un EVI, tomando el éxito del personaje y de los recursos del entorno.

Por esta razón, es conveniente explorar un modelo que permita encontrar un adecuado realismo visual en la percepción y el razonamiento de un personaje en un EVI, debido a que no existe un modelo general reportado en la literatura, los que están, son enfocados hacia una aplicación en específico y no tienen implementado sino solo una de las condiciones de desempeño inteligente, por lo que en este modelo se implementarán dos, que son la percepción y el razonamiento, lo que lleva a un raciocinio cognitivo del personaje. En cuanto a las técnicas de IA utilizadas, solo un trabajo propone el uso de Neuroevolución, mientras que todos los demás están basados en la incorporación de agentes inteligentes. Por esta razón, dentro de las técnicas de IA a utilizar en el modelo propuesto, se pretende no solo emplear agentes inteligentes con el fin de comparar resultados, sino también usar Algoritmos Genéticos y Redes Neuronales, lo que sería un aporte en este campo.

\section{MODELO DE UN PERSONAJE EN UN ENTORNO VIRTUAL INTELIGENTE}

A través del tiempo se ha podido evidenciar cómo han evolucionado la tecnología y las formas de comunicación, incluyendo las novedosas técnicas informáticas como la Realidad Virtual y la IA, que cada vez cogen más auge y abarcan campos diferentes a los relacionados estrictamente con el ámbito tecnológico. Es por eso que deja de ser estrictamente un apoyo para el desarrollo de actividades personales y se convierte en una herramienta de gran utilidad en el desarrollo del campo profesional, incluyendo el entretenimiento y la educación, entre otros. En la última década los entornos virtuales han alcanzado una gran calidad gráfica proporcionada por los adelantos de la tecnología a la par de la capacidad de detalle de los objetos y agentes que lo componen. Hoy en día la investigación centra su atención en el desarrollo de EVI que alcancen grandes capacidades de comportamientos complejos e interactivos para alcanzar un alto nivel de realismo (Cavazza et al., 2005).

En Colombia, se observan entornos virtuales que han sido adaptados de una manera muy básica y que no cuentan con una metodología que permita crear un EVI con cada uno de sus elementos, basados en un estándar que garantice un proceso fluido desde que se idea hasta que se ejecuta. Alrededor del mundo, en universidades, centros de investigación y empresas se encuentran ejemplos de EVI desarrollados sin una filosofía clara que vele por la administración de recursos, la optimización de geometría y la compatibilidad de lenguaje, de máquina y de plataforma, además de brindar parámetros para ubicarlos en una escala análoga al hiperrealismo. Los EVI traen consigo problemas en tiempo real al solucionar sus algoritmos. Ellos dependen de la integración estrecha entre la interactividad natural de un entorno virtual en términos de la visualización centrada en el usuario y la manipulación de objetos, y los aspectos interactivos de la resolución de problemas de algoritmos de IA.

En el ámbito laboral, se necesita el conocimiento, la cooperación y la coordinación de las personas, para que por medio de un trabajo en equipo, desarrollen lo necesario para recrear los entornos en los cuales se van a realizar entrenamientos o inducciones acerca de un trabajo o actividad. Todo eso, al igual que en el campo de la enseñanza, requiere recursos intelectuales, físicos y por ende económicos, los cuales tienden a ser considerados muy altos, precisamente por 
la falta de conciencia sobre la utilidad y los grandes beneficios de los EVI, debido al desconocimiento del tema y a la ausencia de una guía que facilite su implementación en el medio.

Por lo expuesto anteriormente, se hace necesario crear un modelo de referencia para la recreación de personajes inteligentes, apoyados en la percepción y el razonamiento, con el fin de alcanzar un realismo visual, como por ejemplo se puede observar en la Figura 3, que al personaje le pasa corriente cuanto toca un tostador. Esto con el fin de permitir la expansión, conocimiento e implementación de este modelo en diversos campos, debido a que actualmente, las técnicas de simulación por ordenador están adquiriendo cada vez mayor relevancia, debido a su capacidad para optimizar procesos e imitar situaciones o actividades que, por su naturaleza, pueden resultar peligrosas, costosas o entretenidas.

\section{Metodología}

De manera general, la metodología de la presente propuesta, se presenta por etapas a continuación, las cuales cubren el cumplimiento de cada uno de los objetivos propuestos.

Caracterización de objetos y el personaje de un EVI: Lo anterior consiste en definir y describir todos los elementos que intervienen en un EVI, con el fin de seleccionar los relevantes para el diseño del modelo, determinando así la precisión y el alcance de la propuesta.

Diseño del Modelo del EVI: En esta etapa se definirá el nivel gráfico y las herramientas de modelado $3 \mathrm{~d}$ y diseño en $2 \mathrm{~d}$; un $\mathrm{EVI}$ en dos dimensiones difiere en su creación de uno en tres dimensiones, para el primero se pueden utilizar herramientas de diseño típicas ya que solo se manejarían imágenes planas, para el segundo si se requiere una herramienta de modelado $3 \mathrm{~d}$ capaz de exportar la geometría en un formato entendible y manipulable en la plataforma. Lo anterior, lleva a seleccionar los elementos necesarios para tener finalmente el diseño del modelo del EVI de acuerdo a la caracterización realizada en la fase anterior.

Especificación de la técnica de IA a implementar en el modelo de EVI: En esta etapa, se harán comparaciones entre diferentes técnicas de IA, aplicado al comportamiento de objetos y agentes del entorno virtual, con el fin de definir la que pueda garantizar una completa comunicación entre este y los elementos del EVI.

Definir Nivel de Física: En esta etapa se considerarán las características físicas de los elementos del EVI, desde la cinemática hasta los efectos de gravedad, colisión, reacción, etc.

Crear Sistema Semántico: Para la creación del sistema semántico todos los elementos deben ser definidos, asignarles características propias, clasificarlas y definirlas en un contexto universal para la plataforma, es decir que si un elemento del EVI sufre un cambio su entorno virtual circundante lo note.

Validación del modelo propuesto mediante un prototipo: El ajuste de los algoritmos será una etapa esencial para acoplar exitosamente los dos procedimientos propuestos en un proceso final que permita la obtención de un modelo de referencia de un EVI con un adecuado realismo visual. En esta etapa los algoritmos deberán ser modificados hasta encontrar el realismo visual requerido. Se construirá un prototipo computacional para la ejecución de pruebas de éstos algoritmos.

Evaluación de Resultados: Para las pruebas finales se realizará una evaluación del modelo propuesto. Adicionalmente, se evaluará el desempeño temporal entre el modelo propuesto y otros modelos existentes, mediante la realización de un diseño experimental y de pruebas en el prototipo computacional.

\section{CONCLUSIONES}

En esta propuesta, se considera un campo denominado "Entornos Virtuales Inteligentes", el cual está formado por el creciente traslape de las tecnologías involucradas en el desarrollo de gráficos 
interactivos 3d en tiempo real y las técnicas de la Inteligencia Artificial (IA) (Aylett y Cavazza, 2001). Esta propuesta contempla plantear un modelo de personaje inteligente que genere un realismo visual a la hora de interactuar con los elementos en un Entorno Virtual Inteligente. El componente de inteligencia del personaje se proporcionará en la percepción y el razonamiento del mismo, de acuerdo a la técnica que proporcione mejores resultados entre agente inteligentes, redes neuronales y/o computación evolutiva. Una vez escogida la técnica, ésta se implementará en el modelo definido para llegar a un dinamismo entre el personaje y los elementos de un entorno virtual.

\section{REFERENCIAS}

Axling, T, S. Haridi y L. Fahlen. Virtual reality programming in $\mathrm{Oz}$. In:Proceedings of the

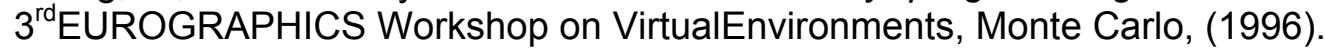

Aylett, RS. y Cavazza, M. Intelligent Virtual Environments - A State-of-the-art Report. Report, Eurographics 2001 - STARs (2001).

Aylett R. y Luck M. Applying artificial intelligence to virtual reality: Intelligent virtual environments. Applied Artificial Intelligence, (2000).

Bee, Nikolauset al. Interacting with a Gaze-Aware Virtual Character.Presented at International IUI 2010 Workshop on Eye Gaze in IntelligentHuman Machine Interaction. (2010).

Bonis, B. et al.A platform for virtual museums with personalized content. Springer Science + Business Media, LLC 2008, (2008).

Calderon, C y Cavazza, M. Intelligent Virtual Environments for Spatial Configuration Tasks.Proceedings of the Virtual Reality International Conference 2001 (VRIC 2001), Laval, France, (2001).

Cavazza et al. Intelligent virtual environments for virtual reality art.Elsevier - Computers \& Graphics 29, 852-861 (2005).

Codognet, P. Animating Autonomous Agents. In: Shared Virtual Worlds,Proceedings of DMS'99, IEEE International Conference on Distributed Multimedia Systems, Aizu, Japan, IEEE Press, (1999).

Codognet, P.Behaviours for virtual creatures by Constraint-based Adaptive Search. In: Working Notes of the AAAISpring Symposium on Artificiallntelligence and InteractiveEntertainment, Stanford, USA, pp. 25-30, (2001).

Decker, Sichman, Sierra, and Castelfranchi.Emotional Input for Character-basedlnteractive Storytelling.Proc. of 8th Int. Conf. on Autonomous Agentsand Multiagent Systems - AAMAS 2009. (2009).

DosSantos, C. y Osorio, F. An Intelligent and Adaptive Virtual Environment and its Application in Distance Learning.AVI '04, May 25-28, 2004, Gallipoli (LE), Italy, (2004).

Edward, L., Lourdeaux, D. y Barth`es, J. Cognitive Modeling of Virtual Autonomous Intelligent Agents Integrating HumanFactors. University of Technology of Compiegne, France.IEEE/WIC/ACM International Joint Conferences on Web Intelligence and Intelligent Agent Technologies. (2009).

Gongora, M e Irvine, D. Adaptive Intelligent Agents based on Efficient Behaviour Differentiation Models.ANDESCON 2010. IEEE. ISBN: 978-1-4244-6740-2. Centre for Comput. Intell., De Montfort Univ., Leicester, UK (2010). 
Guo, A., Zou , Z., Zou , H. y Peng, H. Virtual Actor and its Behavior Simulation Based On Exploratory Analysis. University, Guangzhou,Guangdong, China. Second International Symposium on Knowledge Acquisition and Modeling, IEEE (2009).

Jia, L. y Zhenjiang, M.Entertainment Oriented Intelligent Virtual Environment with Agent and Neural Networks. HAVE 2007, IEEE International Workshop onHaptic Audio Visual Environments and their ApplicationsOttawa, Canada, 12-14 October, (2007).

Latoschik, M. y Fröhlich, C.Semantic Reflection for Intelligent Virtual Environments.IEEE Virtual RealityConference 2007, March 10 -14, Charlotte, North Carolina, USA, (2007).

Lozano, M. y Calderón, C. Entornos Virtuales 3D clásicos e inteligentes: Hacia un nuevo marco de simulación para aplicaciones gráficas 3D interactivas. Journal Inteligencia Artificial, Vol 8, No 23 (2004).

Luzardo, G. y Hernández J. Inteligencia Artificial en Ambientes Virtuales: Humanos Virtuales Autónomos (HVA) como Agentes Virtuales Inteligentes (3DIVA). Disponible en Internet: <http://blog.espol.edu.ec/gluzardo/files/2009/03/trabajo-final.pdf> Consultado en Febrero, 2010, (2010).

Mang-Xian, Q. y Hai-ming, Y. Investigation and Realization of Multi-Agent interaction behavior in Intelligent Virtual Environment.International Conference on Cyberworlds, IEEE Computer Society, (2008).

Mascarenhas, S., Enz, S. y Paiva, A. Using Rituals to Express Cultural Differences in SyntheticCharacters. On Autonomous Agents and Multiagent Systems, AAMAS 2009, Budapest, Hungary, pp. 305-312, (2009).

Silveira, R., Dapper., F., Prestes, E., Nedel, L. Natural steering behaviors for virtual pedestrians. Institute of Informatics, Federal University of Rio Grande do Sul. Vis Comput 26: 1183-1199, (2010).

Tutenel, T. y Bidarra, R. The Role of Semantics in Games and Simulations.ACM Computers in Entertainment, Vol. 6, No. 4, Article 57, Publication date: December 2008, (2008).

Zhong, Z. y Guoxin, T. Intelligent Characters in Virtual Environment. University,Wuhan, Hubei, P.R.China. International Journal of Digital Content Technology and its Applications Volume 3, Number 4, December (2009). 
